\title{
Estimation of prevalence of multidrug resistance in spinal tuberculosis, antibiotic susceptibility patterns and clinical outcomes
}

\author{
Santosh Ghoti, Saurabh Sah*, Sajal Mitra, Amol Bochare
}

Department of Orthopaedics, Government Medical College and Hospital, Nagpur, Maharashtra, India

Received: 17 September 2018

Revised: 13 November 2018

Accepted: 15 November 2018

\author{
*Correspondence: \\ Dr. Saurabh Sah, \\ E-mail: drsaurabhsah@yahoo.com
}

Copyright: (c) the author(s), publisher and licensee Medip Academy. This is an open-access article distributed under the terms of the Creative Commons Attribution Non-Commercial License, which permits unrestricted non-commercial use, distribution, and reproduction in any medium, provided the original work is properly cited.

\begin{abstract}
Background: There are no adequate data in literature referring to the early diagnosis and treatment (especially surgical) of MDR tuberculosis of spine and evaluation of its clinical outcome. We aimed to address this lacunae, by using gene expert test (CBNAAT) and drug sensitivity/susceptibility tests (DST) and by studying subsequent actual clinical outcomes.

Methods: Forty patients with clinico-radiological scenario of spinal tuberculosis were evaluated using biochemical, radiological and histo-pathological studies. Anti-tuberculosis treatment was empirically started based on initial clinico-radiological suspicion of spinal tuberculosis. All tissue specimen and intra-operative samples were subjected to CBNAAT, MGIT culture and histopathological examination. Culture of those samples which showed rifampicin resistance on CBNAAT were followed up for DST (Drug Susceptibility Test) and the treatment modified accordingly, if required. All cases were followed up at 3, 6, 9 and 12 months interval.

Results: Out of 40 patients, mycobacterium tuberculosis was detected in 23 patients by CBNAAT. Out of these 23 cases, 20 showed mycobacterium tuberculosis sensitivity to rifampicin and 3 were resistant. Out of these 3 cases, one had culture positive. Sensitivity and negative predictive value of gene expert test in comparison with the culture were $100 \%$. Specificity and positive predictive value were calculated at $58.6 \%$ and $47.8 \%$ respectively. Accuracy of gene expert in our study was found to be $70 \%$.

Conclusions: Early detection of MDR spinal tuberculosis using highly sensitive CBNAAT test would be helpful to avoid consequences of inappropriate chemotherapy and would result in favourable outcomes.
\end{abstract}

Keywords: MDR-TB, Spinal TB, CBNAAT, Drug susceptibility testing

\section{INTRODUCTION}

One third of the world population is thought to have been infected with mycobacterium tuberculosis, with India contributing nearly $1 / 5^{\text {th }}$ to the total tuberculosis patient burden. ${ }^{1}$ Spinal tuberculosis (Pott's Spine) is one of the oldest diseases. It is the most common extra-pulmonary manifestation of tuberculous infection. ${ }^{1}$

Drug resistance in TB is emerging threat, which has been reported as early as $1948 .^{2}$ But enormity of the problem is being acknowledged only recently. Worse, India ranks second amongst high burden multidrug resistant tuberculosis countries with estimated incidence of $2.3 \%$ MDR-TB (multidrug resistant tuberculosis) cases amongst new cases and 17.2 amongst the previously treated cases. ${ }^{2,3}$ Multidrug resistant tuberculosis (MDRTB) is defined as resistance to Isoniazid and Rifampicin, with or without resistance to other first line drugs (FLD), (WHO 2012). However, the diagnosis and treatment protocol for MDR-TB of spine is not clearly established. There are no adequate studies/data in literature referring 
to the early diagnosis and treatment (especially surgical) of MDR tuberculosis of spine and evaluation of its clinical outcome. ${ }^{3}$

To address these lacunae, this study was undertaken with the objectives of estimation of prevalence of multidrug resistant spinal tuberculosis by using gene expert test (Central based nucleic acid amplification test-CBNAAT), drug sensitivity/susceptibility tests (DST) and studying subsequent actual clinical outcomes.

\section{METHODS}

Study type: Hospital based prevalence study

Study setting: Department of Orthopedics, Government Medical College and Hospital, Nagpur (Tertiary care government centre in central India)

Study duration: 18 months

\section{Inclusion criteria}

Inclusion criteria were patients with clinico-radiological diagnosis of spinal tuberculosis.

\section{Exclusion criteria}

Exclusion criteria were biopsy diagnostic of other pathology such as malignancy etc, refusal to give written consent.

Patients presenting to our centre with suspected spinal tuberculosis were evaluated with $\mathrm{x}$-ray, CT scan, MRI and biochemical test. All patients in whom surgery was not indicated were subjected to percutaneous transpedicular on OPD basis. Biopsy was done under local anaesthesia either fluoroscopy guided or CT guided. Remaining patients with neurological deficit or with indication of surgery were admitted and the biopsy material was collected intra-operatively and sent for investigation.

Anti-tuberculosis treatment was empirically started in all the patients based on initial clinico-radiological suspicion of spinal tuberculosis. All tissue specimens from transpedicular biopsy and intra-operative samples were subjected to CBNAAT, MGIT culture and histopathological examination. Culture of those samples which showed rifampicin resistance on CBNAAT were followed up for DST (Drug Susceptibility Test) and the treatment accordingly modified if required. After the drug sensitivity report for $1^{\text {st }}$ and $2^{\text {nd }}$ line anti-tuberculosis drug was known in rifampicin resistance cases, individualized chemotherapy was formulated on following basis:

- Any $1^{\text {st }}$ line drug to which isolate had proved to be sensitive

- An injectable drug for minimum period of six months
- A Quinolone

- Addition of appropriate second line drug such as Ehionamide and Cycloserine, to achieve combination of four to five effective drug regimen

- Other drug such as Amoxicillin/clavulanate and Clofazimine

- Chemotherapy was ensued for 18-24 month or longer

All cases were followed up at 3 month, 6 month, 9 month and 1 year interval.

Approval from Institutional Ethics Committee was obtained before start of the study. Informed written consent was obtained from each patient before participation in the study. The data was analysed using SPSS (version 20).

\section{RESULTS}

A total of 40 participants were enrolled for the study, out of which one died at home \& cause of his death could not be ascertained. All 40 cases were retained in the final analysis, as relevant.

The mean age of participants was $39.95 \pm 12.78$ years, ranging from 21 to 70 years. $62.5 \%$ (25) of cases were males and $37.5 \%$ (15) were females.

Back pain was universally common complaint seen at presentation in all $(n=40,100 \%)$ cases, followed by constitutional symptoms (evening rise of temperature, loss of weight and loss of appetite) of TB in 28 and neurological-deficit in 23 cases. Thoracic region was most commonly involved $(57.5 \%)$ followed by lumbosacral region (22.5\%); thoracolumbar junction was involved in $17.5 \%$ of cases. Mostly only two vertebrae were affected ( 25 cases), followed by three vertebrae in 9 cases. Out of 40 cases, 6 showed the skip lesion after radiological evaluation.

In most of the cases, tissue samples were collected through fluoroscopy guided transpedicular biopsy (21 cases), followed by collection of tissue samples intraoperatively in 17 cases and in 2 cases samples were obtained by CT guided biopsy. MTB was detected in 12 out of 21 cases $(57.14 \%)$ of fluoroscopy guided transpedicular biopsy; whereas 11 out of 17 samples $(64.71 \%)$ collected intraoperatively were reported positive for MTB. Both the CT guided biopsy samples were reported negative for MTB. Amongst all 40 cases, 20 were managed conservatively and remaining 20 were treated surgically (Anterolateral Decompression with anterior reconstruction for thoracolumbar cases and anterior corpectomy with bone grafting and anterior cervical plating for cervical cases

Out of 40 patients of suspected spinal tuberculosis in our study, mycobacterium tuberculosis was detected in 23 patients by CBNAAT. Out of these 23 cases, 20 showed mycobacterium tuberculosis sensitivity to rifampicin and 
3 were resistant. Out of these 3 cases, only one had culture positive result on which DST performed. MTB growth in that case was resistant to Streptomycin, Ethambutol, Isoniazid, Rifampicin and Ofloxacin and was sensitive to Amikacin, Kanamycin and Capreomycin. Individualised chemotherapy was formulated \& provided as per protocol to the patient. Clinical outcome of this case was excellent till last follow up (18 month) visit.
The result was also good of two cases with rifampicin resistance and culture negative on whom $1^{\text {st }}$ line AKT was continued. Sensitivity and negative predictive value of gene expert test in comparison with the gold standard (culture test) were $100 \%$. But specificity and positive predictive value were calculated at $58.6 \%$ and $47.8 \%$ respectively. Accuracy of gene expert in our study was found to be $70 \%$.

Table 1: Comparison of base-line and 3 monthly interval visual analogue scale (VAS) scores for back pain.

\begin{tabular}{|c|c|c|c|c|c|c|c|c|}
\hline VAS & $\mathbf{F} / \mathbf{u}$ & $\mathbf{N}$ & Mean & SD & $\mathbf{P}$ value & P value* & $\begin{array}{l}\text { Improvement } \\
\text { (each f/u) (\%) }\end{array}$ & $\begin{array}{l}\text { Improvement } \\
\text { (Baseline) }(\%)\end{array}$ \\
\hline \multirow{2}{*}{ Pair 1} & Baseline & 39 & 6.62 & 0.78 & \multirow{2}{*}{$<0.01$} & \multirow{2}{*}{$<0.01$} & \multirow{2}{*}{27.51} & \multirow{2}{*}{27.51} \\
\hline & $3 \mathrm{~m}$ & 39 & 4.80 & 1.38 & & & & \\
\hline \multirow{2}{*}{ Pair 2} & $3 \mathrm{~m}$ & 39 & 4.80 & 1.38 & \multirow{2}{*}{$<0.01$} & \multirow{2}{*}{$<0.01$} & \multirow{2}{*}{31.01} & \multirow{2}{*}{49.99} \\
\hline & $6 \mathrm{~m}$ & 39 & 3.31 & 1.20 & & & & \\
\hline \multirow{2}{*}{ Pair 3} & $6 \mathrm{~m}$ & 22 & 3.59 & 1.18 & \multirow{2}{*}{$<0.01$} & \multirow{2}{*}{$<0.01$} & \multirow{2}{*}{37.98} & \multirow{2}{*}{66.33} \\
\hline & $9 \mathrm{~m}$ & 22 & 2.23 & 1.27 & & & & \\
\hline \multirow{2}{*}{ Pair 4} & $9 \mathrm{~m}$ & 14 & 2.43 & 0.94 & \multirow{2}{*}{$<0.05$} & \multirow{2}{*}{$<0.01$} & \multirow{2}{*}{26.47} & \multirow{2}{*}{73.00} \\
\hline & $12 \mathrm{~m}$ & 14 & 1.79 & 1.31 & & & & \\
\hline \multirow{2}{*}{ Pair 5} & $12 \mathrm{~m}$ & 7 & 2.29 & 1.60 & \multirow{2}{*}{0.172} & \multirow{2}{*}{$<0.01$} & \multirow{2}{*}{12.51} & \multirow{2}{*}{-} \\
\hline & $15 \mathrm{~m}$ & 7 & 2.00 & 1.53 & & & & \\
\hline \multirow{2}{*}{ Pair 6} & $15 \mathrm{~m}$ & 5 & 1.80 & 1.48 & \multirow{2}{*}{0.208} & \multirow{2}{*}{$<0.01$} & \multirow{2}{*}{33.33} & \multirow{2}{*}{ - } \\
\hline & $18 \mathrm{~m}$ & 5 & 1.20 & 0.84 & & & & \\
\hline
\end{tabular}

*Compared from Baseline.

Table 2: Table illustrating progression of ASIA grading over periodic follow up.

\begin{tabular}{|lllllllllllll|}
\hline ASIA & A & & B & & C & D & \multicolumn{1}{c|}{ E } & \multicolumn{3}{c|}{ Total } \\
\hline ASIA & $\mathbf{N}$ & $\mathbf{\%}$ & $\mathbf{N}$ & $\mathbf{\%}$ & $\mathbf{N}$ & $\mathbf{\%}$ & $\mathbf{N}$ & $\mathbf{\%}$ & $\mathbf{N}$ & $\mathbf{\%}$ & $\mathbf{N}$ & $\mathbf{\%}$ \\
\hline Baseline & 5 & 12.8 & 8 & 20.5 & 5 & 12.8 & 4 & 10.2 & 17 & 43.5 & 39 & 100.0 \\
\hline 3 month & 3 & 7.7 & 7 & 17.9 & 7 & 17.9 & 3 & 7.7 & 19 & 48.7 & 39 & 100.0 \\
\hline 6 month & 0 & 0.0 & 2 & 5.1 & 8 & 20.5 & 7 & 17.9 & 22 & 56.4 & 39 & 100.0 \\
\hline 9 month & 0 & 0.0 & 0 & 0.0 & 1 & 4.5 & 5 & 22.7 & 16 & 72.7 & 22 & 100.0 \\
\hline 12 month & 0 & 0.0 & 0 & 0.0 & 0 & 0.0 & 1 & 7.1 & 13 & 92.9 & 14 & 100.0 \\
\hline 15 month & 0 & 0.0 & 0 & 0.0 & 0 & 0.0 & 0 & 0.0 & 7 & 100.0 & 7 & 100.0 \\
\hline 18 month & 0 & 0.0 & 0 & 0.0 & 0 & 0.0 & 0 & 0.0 & 5 & 100.0 & 5 & 100.0 \\
\hline
\end{tabular}

Table 3: Table illustrating sequential improvement in ODI scores over periodic follow up.

\begin{tabular}{|c|c|c|c|c|c|c|c|c|}
\hline ODI & $\mathbf{F} / \mathbf{u}$ & $\mathbf{N}$ & Mean & SD & P value & P value* & $\begin{array}{l}\text { Improvement } \\
\text { (each f/u) (\%) }\end{array}$ & $\begin{array}{l}\text { Improvement } \\
\text { (Baseline) (\%) }\end{array}$ \\
\hline \multirow{2}{*}{ Pair 1} & Baseline & 39 & 65.79 & 16.07 & \multirow{2}{*}{$<0.01$} & \multirow{2}{*}{$<0.01$} & \multirow{2}{*}{24.22} & \multirow{2}{*}{24.22} \\
\hline & $3 \mathrm{~m}$ & 39 & 49.86 & 23.34 & & & & \\
\hline \multirow{2}{*}{ Pair 2} & $3 \mathrm{~m}$ & 39 & 49.86 & 23.34 & \multirow{2}{*}{$<0.01$} & \multirow{2}{*}{$<0.01$} & \multirow{2}{*}{23.46} & \multirow{2}{*}{42.00} \\
\hline & $6 \mathrm{~m}$ & 39 & 38.16 & 21.82 & & & & \\
\hline \multirow{2}{*}{ Pair 3} & $6 \mathrm{~m}$ & 22 & 42.05 & 20.29 & \multirow{2}{*}{$<0.01$} & \multirow{2}{*}{$<0.01$} & \multirow{2}{*}{38.06} & \multirow{2}{*}{60.41} \\
\hline & $9 \mathrm{~m}$ & 22 & 26.05 & 15.21 & & & & \\
\hline \multirow{2}{*}{ Pair 4} & $9 \mathrm{~m}$ & 14 & 24.79 & 9.73 & \multirow{2}{*}{$<0.05$} & \multirow{2}{*}{$<0.01$} & \multirow{2}{*}{21.04} & \multirow{2}{*}{70.25} \\
\hline & $12 \mathrm{~m}$ & 14 & 19.57 & 10.29 & & & & \\
\hline \multirow{2}{*}{ Pair 5} & $12 \mathrm{~m}$ & 7 & 20.00 & 9.31 & \multirow{2}{*}{0.231} & \multirow{2}{*}{$<0.01$} & \multirow{2}{*}{5.72} & \multirow{2}{*}{-} \\
\hline & $15 \mathrm{~m}$ & 7 & 18.86 & 10.06 & & & & \\
\hline \multirow{2}{*}{ Pair 6} & $15 \mathrm{~m}$ & 5 & 18.40 & 10.90 & \multirow{2}{*}{0.103} & \multirow{2}{*}{$<0.01$} & \multirow{2}{*}{21.74} & \multirow{2}{*}{-} \\
\hline & $18 \mathrm{~m}$ & 5 & 14.40 & 7.92 & & & & \\
\hline
\end{tabular}

*Compared from baseline 
Out of 40 cases, 18 showed histopathological signs of chronic granulomatous changes in favour of tuberculous pathology and rest of the 22 cases the histopathological report was inconclusive.

Functional and clinical outcome was evaluated by Visual Analogue Scale (VAS), ODI and ASIA grading. At baseline, average VAS scores were in the range of 6-7. Improvements in VAS score as compared with baseline at the each subsequent follow up were significant up to 18 months. When VAS score was compared with previous follow up visit VAS score, significant improvement was noted up to 12 months. Maximum improvement in VAS score occurred in first 6 months of follow up (50\%) (Table 1).

As for ASIA (American Spinal Injury Association) score, maximum numbers of cases were in the grade $\mathrm{E}(17$ cases, $42.5 \%$ ), followed by grade B (9 cases, $22.5 \%$ ), 5 cases each in Grade A and Grade C, rest of the 4 cases were in Grade D. At 6 months follow-up, out of 39 cases, 22 were in grade E, 7 cases in grade D, 8 cases in grade C and only one case in grade B. Significant improvement was noted in neurological status of the cases over the time (Table 2).

When Oswestry Disability Index (ODI) was calculated, at baseline, maximum number of cases were in the severe disability $42.5 \%$ (17 cases), $32.5 \%$ (13 cases) were in crippled category and rest of $25 \%$ (10 cases) cases were bed ridden. There was significant improvement in ODI score as compared with baseline ODI score over each follow up visit up to 18 months. If ODI score was compared with previous follow up, then improvement was significant up to 12 months. Maximum improvement in VAS score as compared with baseline VAS score occurred in $1^{\text {st }} 9$ months follow up (70\%) (Table 3).

When ESR was estimated, maximum numbers of cases were in the range of $20-60 \mathrm{~mm}$ at the end of $1 \mathrm{hr}$. There was significant improvement in ESR over subsequent follow up visit up to 6 month. The mean baseline ESR improved from $49.59 \pm 18.64$ to $21.72 \pm 10.66 \mathrm{~mm}$ at the end of $1 \mathrm{hr}$.

\section{DISCUSSION}

Prevalence of spinal tuberculosis in India has been reported to be $1-5 \%$. There are no clear guidelines regarding type and duration chemotherapy for spinal tuberculosis. Compounding that problem is the rise of MDR-TB. Also there are no guidelines to suspect that a patient may be having MDR-TB. In order to the assertion the prevalence of MDR-TB in patients of spinal tuberculosis presenting to our institute and to assertion the positivity of performing tissue biopsy in every patient and its benefits, this study was undertaken.

We performed fluoroscopic guided transpedicular biopsy in 21 out of 40 patients $(52.5 \%)$ under local anaesthesia,
CT guided biopsy in 2 patients and in 17 patients samples were collected intraoperatively. Out of 21 patients, Mycobacterium tuberculosis was detected by CBNAAT in 12 patients $(57.14 \%)$, however M-TB detected in 19 patient by Shrestha et al in 23 cases. $^{4}$ In our study we have started empirical AKT in all patients. We have performed tissue biopsy in patients after the empirical AKT, which could be the reason for low positivity in our study. In 2 patients we have performed CT guided biopsy, but in both tissue sample MTB was not detected.

Pain was assessed using VAS with mean baseline score of $6.6 \pm 0.77$. There was significant improvement in the VAS score for the back pain over months. The improvement in the VAS score showed consistent pattern, with improvement being significant at each follow up visit up to 12 months. The maximum improvement in VAS scores were in $1^{\text {st }} 6$ months $(50 \%)$. Qing-Yi et al reported similar improvement in VAS score in their study of treatment effect and postoperative complication in 54 patient of thoracic and lumbar spinal tuberculosis. ${ }^{5}$ In their study VAS score of 7.8 \pm 1.7 improved to $3.2 \pm 2.1$ at the last follow up.

There was significant improvement in the ODI score for disability arising as a consequence of the back pain. The improvement in disability score was consistently significant at each follow up visit up to 12 month, suggestive of progressive decrease in the disability due to the back pain. The mean ODI score at the baseline $(65.79 \pm 16.07)$ improved to $38.16 \pm 21.82$ at 6 month follow up of all patients. The result are comparable to a study by Huang et al, in which they evaluated the clinical outcomes of surgical treatment of non-contagious spinal tuberculosis in 23 cases. $^{6}$ In their study, mean ODI improved from 52.57 before surgery to 25.36 at the last visit after 12 months.

The American Spinal Injury Association (ASIA) score was used to evaluate neurological deficit. Significant improvement was noted in neurological status of the cases over the time, when patients were studied every 3 months for a total 18 of months follow up. This was comparable to study conducted by Sharma et al. ${ }^{7}$ This study result was also comparable to study conducted by $\mathrm{Li}$ et $\mathrm{al}^{8}{ }^{8}$ On retrospective analysis of management of drug resistant spinal tuberculosis in 35 patients, improvement was noted in ASIA scores, from 17 cases with Grade E at initial presentation to 29 Cases with Grade $\mathrm{E}$ at the final follow up visit. A major limitation w.r.t. is that a significant chunk of our patients were lost to follow up, with only 5 patients returning for ASIA evaluation at 18 months.

In our study ESR showed significant improvement up to 6 month. This was comparable with study conducted by Huang et al. ${ }^{6}$ Literature suggests histologic studies to confirm the diagnosis of spinal tuberculosis in approximately $60 \%$ of patients. ${ }^{6}$ In our study histopathological examination of samples showed result 
in favour of spinal tuberculosis in $65 \%$ patients. These results were also comparable with study conducted by Tuli et al, who reported positive histological finding in favour of spinal tuberculosis in $60 \%$ of evaluated cases. ${ }^{9}$ With the advent of PCR method to test for TB, various studies have reported the sensitivity of non-automated PCR test to be ranging from $61-83 \% .^{10-14}$ The PCR method has the advantages of needing much smaller quantities of mycobacterium tuberculosis and giving rapid diagnosis by amplification of nucleic acid of the pathogen. Its detection limit of 130 colony-forming units (CFU) per millilitres, compared with $10,000 \mathrm{CFU} / \mathrm{ml}$ in cultures, increase the probability of diagnosing extrapulmonary tuberculosis even with samples containing only a few bacteria. In our study sensitivity and specificity of CBNAAT test were reported at $100 \%$ and $58.8 \%$ respectively. The prevalence rate of multidrug resistance spinal tuberculosis in our study was $2.5 \%$ (1 out of 40 patients). This was comparable with rate of multidrug spinal tuberculosis in new cases $(2.3 \%)$ by World Health Organization. ${ }^{15}$ Furthermore, rate of Rifampicin resistance was $7.5 \%$ in our study (3/40). This was comparable to a study by Sharma et al. ${ }^{7}$ Their noted rate of Rifampicin resistance was $4.8 \%$ (7/145). CBNAAT parameters in our study were sensitivity$100 \%$, specificity-58.6\%, PPV-47.8\%, NPV-100\% and accuracy of $70 \%$. Kumar et al reported the sensitivity and specificity of gene expert test at $94 \%$ and $64 \%$ respectively. Sharma et al reported sensitivity of gene expert test at $93.4 \% .^{7}$ Held et al reported the sensitivity as well as specificity of gene xpert test to be as high as $95.6 \%$ and $96.2 \%$ respectively. $^{16}$

One of the disadvantages of CBNAAT (PCR) test is that, in contrast to TB culture, gene expert testing will give a positive result even if the pathogen is not viable. ${ }^{17}$ In these patients, active spinal tuberculosis has to be confirmed clinically and by means of various imaging modalities. Another concern about gene expert method is that it tests drug resistance only for Rifampicin; therefore mono resistance to Isoniazid can only be detected with other tests. According to current treatment guidelines Isoniazid mono-resistance does not alter conventional TB regime for patients. Other PCR tests like line probe assay detect resistance simultaneously for Isoniazid and Rifampicin; but sensitivity and specificity for extrapulmonary tuberculosis detection of CBNAAT test is more as compared with line probe assay. Line probe assay is technically more difficult, takes longer time (5 day), and is carried only on smear positive samples. Extra-pulmonary samples are mostly pauci-bacillary and smear negative. ${ }^{10}$ Hence its utility seems limited in the given set up.

In conclusion, early detection of MDR Spinal Tuberculosis would be helpful to avoid consequences arising due to in appropriate chemotherapy and, hence, would also result in favourable outcomes. We report high sensitivity of CBNAAT test in detection of mycobacterium Tuberculosis with the added advantage of almost immediate results. We recommend its use in therapeutically refractory cases, cases where the imaging is not as typical of Potts spine, and in patients undergoing surgery, as no separate procedure is required for obtaining the tissue sample. However, further similar studies with larger samples are needed before we can make definitive statement about the usage of CBNAAT Test as routine diagnostic tool in suspected MDR Spinal Tuberculosis cases.

\section{Funding: No funding sources}

Conflict of interest: None declared

Ethical approval: The study was approved by the institutional ethics committee

\section{REFERENCES}

1. Taylor GM, Murphy E, Hopkins R. First report of mycobacterium tuberculosis bovis DNA in human remain from iron-age. 2007;153(4):1243-9.

2. Jain AK, Dhammi IK. Tuberculosis of the spine:A review. Clinorthop Related Res. 2007;460(July):3949.

3. Jain AK, Dhammi IK, Modi P, Kumar J, Sreenivasan R, and Saini N. Tuberculosis spine:Therapeutically refractory disease. Indian $\mathrm{J}$ Orthop. 2012;46(2):171-8.

4. Shrestha D, Shrestha R, Dhoju D. Fluoroscopy Guided Percutaneous Transpedicular Biopsy for Thoracic and Lumbar Vertebral Body Lesion:Technique and Safety in 23 Consecutive Cases. Kathmandu Univ Med J. 2015;51(3):256-60.

5. He QY, Xu JZ, Zhou Q, Luo F, Hou T, Zhang Z. Treatment effect, postoperative complications, and their reasons in juvenile thoracic and lumbar spinal tuberculosis surgery. J Orthop Surg Res. 2015;10:156.

6. Huang J, Zhang H, Zeng K, Gao Q. The clinical outcome of surgical treatment of noncontagious spinal tuberculosis: A Retrospective study in 23 cases. PLos One. 2014;9(4):e93648.

7. Sharma A, Chhabra HS, Mahajan R, Chhabra T, Batra S. Magnetic Resonance Imaging and GeneXpert: A Rapid and Accurate Diagnostic Tool for the Management of Tuberculosis of the Spine. Asian Spine J. 2016;10(5):850-6.

8. Litao Li, Zehua Zhang, Fei Luo, Jianzhong $\mathrm{Xu}$, Peng Cheng, Zheng Wu et al. Management of drugresistant spinal tuberculosis with a combination of surgery and individualised chemotherapy:a retrospective analysis of thirty-five patients Int Orthop (SICOT). 2012;36:277-83.

9. Tuli SM. Tuberculosis of the skeletal system:bones, joints, spine and bursal sheaths. New Delhi:Jaypee Brothers Pvt. Ltd.; 2010.

10. Lawn SD, Nicol MP. Xpert@ MTB/RIF assay: development, evaluation and implementation of a new rapid molecular diagnostic for tuberculosis and rifampicin resistance Future Microbiol. 2011;6(9):1067-82. 
11. Suzana S, Ninan MM, Gowri M, Venkatesh K, Rupali P, MichaelJS. Xpert MTB/Rif for the diagnosis of extrapulmonary tuberculosis--an experience from a tertiary care centre in South India. Trop Med Int Health. 2016;21(3):385-9.

12. Scott LE, Beylis N, Nicol MP, Nkuna G, Molapo S, Berrie L, et al. Diagnostic Accuracy of Xpert MTB/RIF for Extrapulmonary Tuberculosis Specimens: Establishing a Laboratory Testing Algorithm for South Africa J ClinMicrobiol. 2014;52(6):1818-23.

13. Lawn SD, Zumla A. Diagnosis of extrapulmonary tuberculosis using the Xpert MTB/RIF assay. Anti Infect Ther. 2012;2012:631-5.

14. World Health Organization 'Automated real-time nucleic acid amplification technology for rapid and simultaneous detection of tuberculosis and rifampicin resistance: Xpert MTB/RIF assay for the diagnosis of pulmonary and extrapulmonary TB in adults and children'. Available at: http://www.who.int/about/licensing/copyright_form/ en/index.html. Accessed on 5 September 2018.

15. The World Health Organization. Union against tuberculosis: Global project on anti-tuberculosis drug resistance surveillance. Fourth Global Report. 2002-2007.

16. Held M, Laubscher M, Zar HJ, Dunn RN. GeneXpert polymerase chain reaction for spinal tuberculosis. Bone Joint J. 2014;96-B:1366-9.

17. Kumar M, Kumar R, Srivastava AK, Nag VL, Maurya AK, Dhole TN, et al. Sensitivity of PCR IS6110 in relation to culture and staining in Pott's disease. Indian J Neurosurg. 2013;2:46-51.

Cite this article as: Ghoti S, Sah S, Mitra S, Bochare A. Estimation of prevalence of multidrug resistance in spinal tuberculosis, antibiotic susceptibility patterns and clinical outcomes. Int J Res Orthop 2019;5:115-20. 